\title{
SOME MODELS OF RASTER CORRELATORS OF BINARY IMAGES
}

\author{
Ruslan R. Zholtikov, Mikhail M.Tatur \\ Computer Department, Belarusian State University of Informatics and Radioelectronics. \\ 6, Brovka str., Minsk, 220027, Belarus. \\ e-mail: tatur@bsuir.unibel.by.
}

\begin{abstract}
In paper the outcomes of mathematical modeling of statistical recognition of binary images are proposed. The offered hypothesis that the pixels constituting boundary of recognition object and an image background are secondary attributes at recognition is experimentally confirmed. As consequence, recognition reliability can be raised due to exception of these pixels at recognition. Basing on the offered example of training of models on the fixed training sample and taking into account that the prototype model is a special case of the rejecting model we have noted that recognition reliability of offered model cannot be lower than reliability of the prototype. The obtained results will be used in hardware realization of binary comparators.
\end{abstract}

Keywords: pattern recognition, binary raster, raster correlators, fuzzy boundary

\section{INTRODUCTION}

The model of the binary statistical correlator, as pattern classifier, is the object of our research [1]. As the prototype the classical model consisting of the binary raster comparator and threshold unit have been examined. Its structure is represented on fig.1.

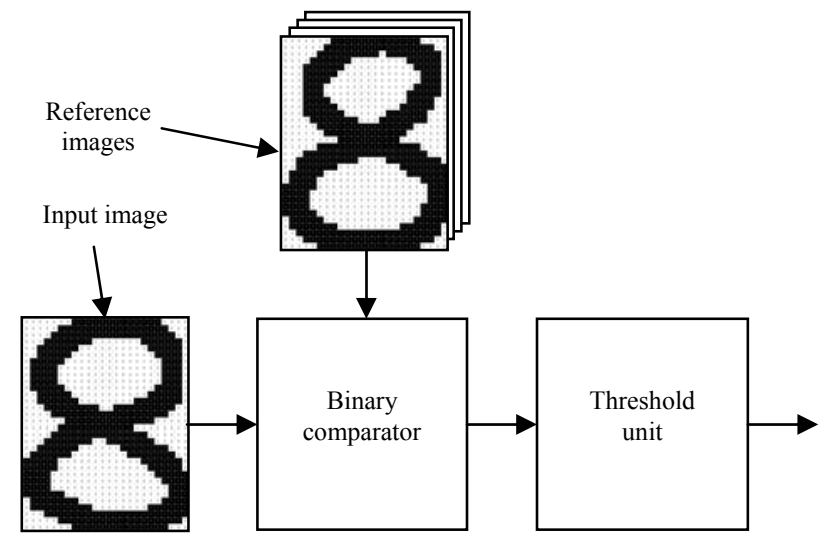

Fig.1. Initial model of the raster correlator.

The comparator carries out bit-by-bit comparison of the input image and a series of the reference images and calculates logic $S_{j}^{a b s}$ for each class.

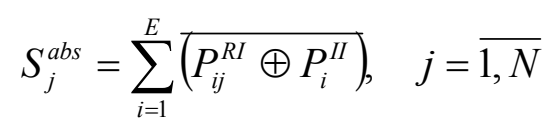

where $P_{i}^{I I}-i^{\text {th }}$ pixel of the input image, $P_{i j}^{R I}-i^{\text {th }}$ pixel of the $j^{\text {th }}$ reference image, $E$ - size of a binary raster, $N$ - quantity of classes.
The preference about the input image is given to that class where there were a maximal total number of pixels concurrences. Function of the threshold unit has the following kind:

$$
X=J\left\{\max \left(S_{j}^{a b s}\right)\right\}, j=\overline{1, N}
$$

As a rule, raster correlators are used when quantity of classes is insignificant, for example, at recognition of alphabet characters, road signs, national emblems and etc. Input images should meet the certain requirements. They should be normalized on scale, oriented in a matrix and separate fragments of input images should be proportionate. Thus, restrictions to the input images are formulated as in the majority of statistical methods, for example, spectral or neuron-like.

Main problem of these methods is the learning. For researched model of raster correlators is a reference images formation. Obviously, recognition quality will be caused by a discrepancy degree of the same input and reference images. Thus, the greatest probability of pixels discrepancies will belong to the object boundary.

The working hypothesis will be that the pixels located on object boundary practically do not carry the information on an image. Moreover, such pixels account reduces recognition probability. Hence, reliability of recognition can be raised at the exception of these pixels at recognition. Thus it is necessary to solve a problem of optimum learning. 
The purpose of given article is experimental confirmation of this hypothesis.

\section{LEARNING OF BINARY CORRELATORS}

As optimum learning of correlators we shall understand a finding of such reference images which would provide recognition of the maximal number of input images. Learning can be divided into two stages[2].

On the first stage, for each class, with using of final set of learning images, the so-called statistical account of class (SAC) is computing. SAC is a matrix of the data of size E. Each SAC element value determines quantity of the object pixels in learning images.

$$
S_{i j}^{S A C}=\sum_{k=1}^{M_{j}} P_{i j k}^{L I}, \quad i=\overline{1, E}, j=\overline{1, N}
$$

where $S_{i j}^{S A C}-i^{\text {th }}$ element of the static account for class $j, P_{i j k}^{L I}-i^{\text {th }}$ pixel of $k^{\text {th }}$ learning image for class $j, M_{j}$ - quantity of learning images for class $j$.

At the second stage of learning it is necessary to determine reference images basing on obtaining SACs.

For the initial model (1), the task of reference images determination is reduced to a finding of optimum boundary of the object and background [3]. On fig. 2 qualitative dependence of probabilities distribution of $i^{\text {th }}$ pixel belonging to a background of the image (solid line) and object of recognition (dashed line) is submitted depending on $S_{i j}^{S A C}$.

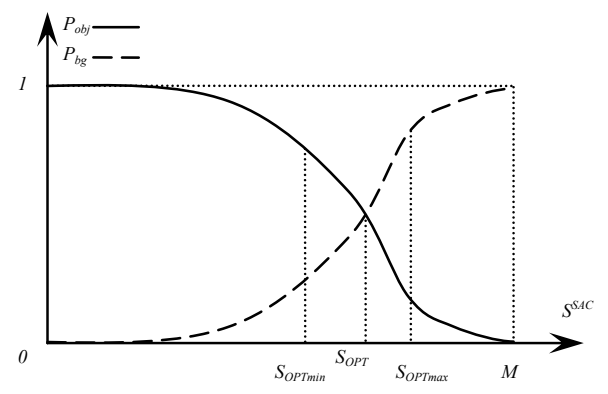

Fig. 2 Probabilities distribution of object (background) pixel belonging

The crossing point of two lines characterizes an optimum threshold $\left(S_{O P T}\right)$ of the background and object boundary. Then

$$
P_{i j}^{R I}=\left\{\begin{array}{l}
1, S_{i j}^{S A C}>S_{O P T} \\
0, S_{i j}^{S A C} \leq S_{O P T}
\end{array}\right.
$$

Developing a working hypothesis, we assume, that there is a pair thresholds $S_{O P T \min }$ and $S_{O P T \max }$ which using will allow us to allocate a subset of boundary pixels. These pixels exception(rejection) at recognition finally should raise recognition efficiency. Thus, in the given approach it is necessary to determine for each class as the reference image, and rejection mask. Reference image and rejection mask pixels will be defined according to expressions:

$$
\begin{gathered}
P_{i j}^{R I}=\left\{\begin{array}{l}
1, S_{i j}^{S A C}>S_{O P T \max } \\
0, S_{i j}^{S A C} \leq S_{O P T \min }
\end{array}\right. \\
P_{i j}^{R M}= \begin{cases}1, & S_{i j}^{S A C} \leq S_{O P T \min }, S_{i j}^{S A C}>S_{O P T \max } \\
0, & S_{O P T \text { min }}<S_{i j}^{S A C} \leq S_{O P T \max }\end{cases}
\end{gathered}
$$

\section{REJECTION MODEL OF THE RASTER CORRELATOR}

On fig. 4 it is submitted structure of rejection model of the raster correlator.

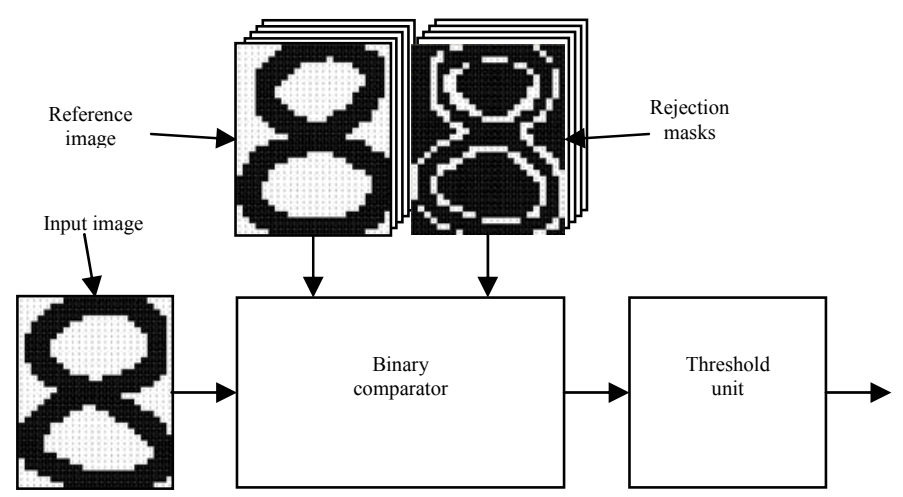

Fig.4. Rejection model of the raster correlator.

Alongside with $\mathrm{N}$ reference images, in formation of the sums $S_{j}^{r}$ participate $\mathrm{N}$ rejection masks. Functioning of binary comparator is described by the following expression:

$$
S_{j}^{r}=\sum_{i=1}^{E}\left(\overline{\left.P_{i j}^{R I} \oplus P_{i}^{I I}\right)} \& P_{i j}^{R M}, j=\overline{1, N}\right.
$$

where $P_{i}^{I I}-i^{t h}$ pixel of the input image, $P_{i j}^{R I}-$ $i^{\text {th }}$ pixel of the $j^{\text {th }}$ reference image, $P_{i j}^{R M}-i^{\text {th }}$ pixel of the $j^{\text {th }}$ rejection mask, $E$ - size of a binary raster, $N-$ quantity of classes.

At exception of some pixels set, the number of pixels participating in recognition for different classes can be various. Thus, the choice of the maximal concurrence is possible only at operating 
by relative values of total number of pixels coincidences.

$$
S_{j}^{r e l}=\frac{S_{j}^{r}}{\sum_{i=1}^{E} P_{i j}^{R M}}
$$

The preference about the recognized image is given that class where there were a maximal relative number of coincidences. The threshold unit realizes the same function (2), but with a relative values:

$$
X=J\left\{\max \left(S_{j}^{r e l}\right)\right\}, j=\overline{1, N}
$$

\section{EXPERIMENTAL CONFIRMATION OF A WORKING HYPOTHESIS}

Researches were spent on the model realizing recognition of hand-written Arabian numbers.

Reference images formation was carried out basing on of 25 binary learning images for each class. Theirs size equals $24 \times 32$ a pixels. In total 250 input images participated in experiment (25 images for each class).

For the initial correlator at each optimal threshold and for the rejection correlator at various combinations of $\min$ and $\max$ thresholds the recognition probability was calculated. Results of experiment are submitted on fig.5.

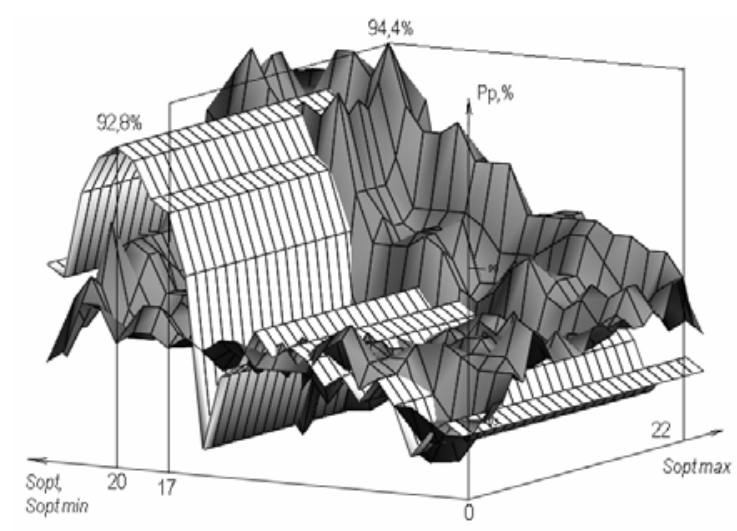

Fig.5. Recognition probability distribution with various thresholds for both models

Apparently from fig.5 for the initial model (white graph) an optimum threshold $S_{O P T}=20$. At the given threshold the correlator has correctly recognized 232 images, and the recognition probability has come to $92,8 \%$.

For rejection correlator optimum there was a pair of thresholds $S_{\text {OPTmin }}=17$ and $S_{\text {OPTmax }}=22$. In this case it has been recognized 236 of 250 submitted images and recognition probability has come to 94,4 $\%$.

In experiment for revealing the reasons causing increase of recognition efficiency additional characteristics have been obtained.

At correct recognition of the image were calculated: total number of pixels coincidences of the input and reference images for that class which has a maximum total number coincidences; difference between total number of pixels coincidences for the two classes which have the most coincidences

Average values of those characteristics are resulted in tab. 1.

Table 1. Experimental outcomes

\begin{tabular}{|l|c|c|}
\hline & Initial model & $\begin{array}{c}\text { Rejection } \\
\text { model }\end{array}$ \\
\hline$S_{\max } \%$ & 84,753 & 85,666 \\
$S_{\max 0}-S_{\max 1}, \%$ & 11,808 & 12,203 \\
\hline
\end{tabular}

Increase of recognition efficiency is connected with improvement of "quality" of reference images. Excluding fuzzy boundary pixels from consideration, recognition is carried out on set of more informative attributes. Thus fuzzy boundary pixels inhibit recognition reducing number of pixels coincidences for correct class and increasing it for others.

For revealing dynamics of decrease of recognition quality at increase of class number the following experiment has been carried out. For the classes set consisting 26 hand-written Latin alphabet symbols and 10 Arabic numbers, the reference images for the prototype-model and pair of the reference images and rejecting masks for offered model have been obtained. Then both models made recognition among 2, 3 and so on up to 36 classes. For each quantity of classes participating at recognition the relative recognition probabilities for both models were calculated. The test sequence contains on 35 samples for each class, in total 1260 images. Results of experiment are represented on fig. 6.

Apparently from fig. 6 with increase in quantity of classes the percent of correctly recognized images decreases, but reduction occurs not so quickly at using rejecting model. For example, at 5 classes of images the difference equal $1,4 \%$, and at 36 classes the rejecting model advantage already 3,6\%. This 
experiment once again confirms advantage of use of offered rejecting model.

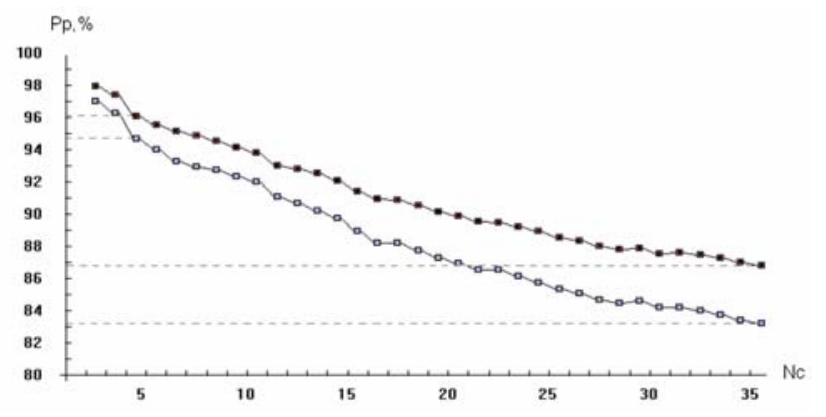

Fig.6. Recognition probability distribution with various numbers of classes at recognition.

\section{CONCLUSION}

It is considered to be, that increase of efficiency is connected to a spent resource with nonlinear dependence. In the resulted example, increase of recognition probability has demanded double increase in a memory size. As a whole, the degree of increase of recognition probability depends on many factors: learning quality, width of learning set, number input images and, finally, from statistical characteristics of object and model of recognition.

It is necessary to note that in a worst case when $\mathrm{S}_{\mathrm{OPT} \min }=\mathrm{S}_{\mathrm{OPT} \min }=\mathrm{S}_{\mathrm{OPT}}$, and all pixels of rejection masks are true rejection model transformed to classical initial model. Hence, recognition reliability of rejection model of the raster correlator, by definition, cannot be lower than reliability of initial model. Thus, the working hypothesis about increase of recognition probability of raster correlators proves to be true at the expense of fuzzy boundary of recognition objects.

The obtained results can be used for hardware realization of binary processing devices.

\section{REFERENCES}

[1] William K. Pratt. Digital image processing: PIKS Inside, $3^{\text {rd }}$ Edition, John Wiley \& Sons; 2001.

[2] M.J.B. Duff, T.J. Fountain. Cellular Logic Image Processing, London, Academic Press, 1986, p.278.

[3] H.R. Tizhoosh, B.M. Otto-von-Guericke Improvement of image quality based on subjective evolution and fuzzy aggregation techniques, Proc. EUFIT'98, Aachen, Germany, 1998, v.2,1325-1329

[4] A.Yu. Lutsyk, B.V. Kisil, O. N. Perenskyy. Image processing in real time on configurable computing architecture, Proc. $3^{\text {rd }}$ International Conference "Digital Information processing and control in extreme situation", Minsk, Belarus, 2002, pp 57-62.
[5] P.V.Akulich, M.V. Grigor'ev, and others. Intelligent decipherer of binary images. Proc. $6^{\text {th }}$ ISTC SACS "Science and Computing", Moscow, Russia, 2003, p.104

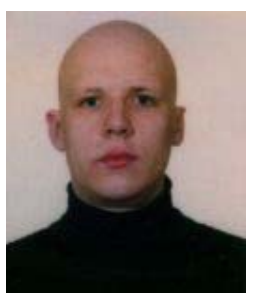

Ruslan R. Zholtikov is postgraduate student of Belarusian state university of informatics and radioelectronics. In 2001 has graduated from this university. In this year has entered to the postgraduate school. Has published 10 scientific works. Main areas of scientific exploration are image processing and pattern recognition and fuzzy logic.

Mikhail M.Tatur is senior lecturer in Belarusian state university of informatics and radioelectronics. In 1981 has graduated from Military academy of Belarus. In 1986 has defended an academic degree of the candidate of sciences in technical diagnosis. Has published 90 works, including monograph, 30

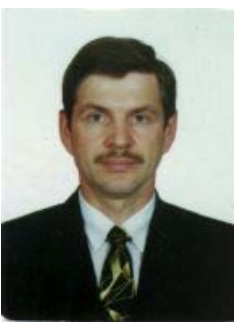
inventions and 15 articles. Main areas of scientific exploration are technical diagnosis, image processing and pattern recognition, fuzzy logic and design of the multiple-processor regular systems 